\title{
Adaptive fusion localization mechanism towards TDoA and IMU data with LSTM correction Method
}

\author{
$1^{\text {st }}$ Zhiyuan $\mathrm{Liu}^{1}, 2^{\text {nd }}$ Liang $\mathrm{Li}^{2}, 3^{\text {nd }}$ Zheng Wang ${ }^{3}, 4^{\text {nd }}$ Chen $\mathrm{Ao}^{4}, 5^{\text {nd }}$ Qiang $\mathrm{Fu}^{5}$, \\ $6^{\text {nd }}$ Puning Zhang ${ }^{6}$ \\ \{liuzhiyuan@nx.sgcc.com.cn'1,liliang1@sgitg.sgcc.com.cn²,wangzheng3@sgitg.sgcc.com.cn ${ }^{3}$,aochen@s \\ gitg.sgcc.com.cn ${ }^{4}$,fuqiang1@sgitg.sgcc.com.cn ${ }^{5}$,zhangpn@cqupt.edu.cn 6 \} \\ Maintenance Filiale of State Grid Ningxia Power Co., Yinchuan 750011,Ningxia, China ${ }^{1}$ \\ State Grid Key Laboratory of Power Industrial Chip Design and Analysis Technology, Beijing Smart- \\ Chip Microelectronics Technology Co., Ltd. Beijing 100192, China ${ }^{2,3,4,5}$, \\ Chongqing University of Posts and Telecommunications, \\ No. 2 Chongwen Road, Nan 'an District, Chongqing, China ${ }^{6}$
}

\begin{abstract}
Using Time Difference of Arrival(TDoA) positioning results and the Inertial measurement unit(IMU) for calculating the motion state of information fusion can significantly improve the positioning accuracy, due to the carrier in the process of movement, the state of the system noise and measurement noise are not strictly obey the normal gaussian distribution, which makes the traditional fusion positioning method using Kalman Filtering algorithm less accurate. This paper proposes an adaptive filter fusion localization mechanism with LSTM network correction. Firstly, a data preprocessing method is designed to convert IMU data from the carrier coordinate system to the geographical coordinate system. Then, based on kinematics theory, the state equation and measurement equation of Adaptive Kalman Filter are established and the system state noise is obtained. Furthermore, the model adaptively to update the carrier coordinate, system state noise and measurement noise. Finally, the carrier trajectory coordinates predicted by the coupled LSTM model are used to obtain the final positioning results and complete the carrier trajectory filtering. Experimental results show that the proposed fusion localization mechanism can effectively improve the accuracy of carrier trajectory localization.
\end{abstract}

Keywords: Fusion positioning; TDoA; IMU; Adaptive Kalman Filter; LSTM

\section{Introduction}

In recent years, with the continuous developments of location technology, Location-Based Service (LBS) has received more and more attentions. LBS is an indispensable foundation and support for many technologies such as mobile terminals, the Internet of Things, and virtual reality. Intelligent warehousing and intelligent transportation in the Internet of Things, somatosensory games and human-computer interaction in virtual reality, navigation services and data statistics in mobile terminals all put the acquisition and perception of LBS in an indispensable part, which shows broad business prospects and huge market value ${ }^{[1]}$. As the development of modern science and technology, information query services such as eating, drinking and playing, as well as LBS services, such as accidental location service, have urgent requirements on positioning accuracy. If the positioning deviation is too large, the application 
value will be lost. In the current research, LBS is combined with user demand information to expand the broader development prospects and application space, such as navigation and tracking, location data fusion, and sniper marketing, etc.

For substations, a single positioning method is difficult to meet the needs of complex substation environments, so multiple positioning methods need to be used for joint positioning. The existing smart phone terminal is equipped with many sensor components, and their IMU is inexpensive. So, based on TDoA positioning, the use of IMU for joint assisted positioning has become a new trend. In literature [2], a combined positioning algorithm for indoor TDoA is proposed to work in the case of normal line of sight signals. In the case of signal interference, the IMU is used to provide accurate positioning in a short time to assist the TDoA algorithm in positioning, and the Kalman filter is used for data preprocessing. In literature [3], the author uses the TDoA system and an IMU consisting of an accelerometer to estimate the position of the pedestrian, and performs extended Kalman filtering on the measurement of the IMU to obtain the number of user's step, step size and fast switching of the user direction. In literature [4], under the influence of non-line of sight (NLOS) error in TDoA measurement to mobile platform, a precision distance difference selection method based on IMU measurement was developed, which was combined with iterative extended kalman filter to effectively reduce NLOS error. Both the Kalman filter and the extended Kalman filter algorithm used in the existing literature assume that the noise of the positioning system obeys the Gaussian distribution of the mean of $0^{[6-9]}$. But in real life, the system noise changes constantly due to the up and down jitter of the measurement personnel during the movement and the noise of the equipment. Therefore, it is necessary to propose a filtering method that can adapt the complex noise of the system to improve the positioning accuracy.

Aiming at the low prediction accuracy and poor scalability of traditional models, this paper proposes an adaptive filtering fusion localization mechanism with LSTM network correction. Firstly, a data preprocessing method is designed to pre-filter the data of the inertial measurement unit and convert the IMU data from the carrier coordinate to the geographic coordinate. Then, based on the kinematics theory, the state equation of the system is constructed and the state noise of the system is obtained. Furthermore, the adaptive Kalman filter algorithm is used to adaptively update the carrier coordinates, system state noise, and measurement noise. Finally, the LSTM model is adopted to predict the carrier trajectory coordinates, the weighted average of the prediction results and the adaptive filtering results are used to achieve the fusion positioning of the carrier.

\section{System structure}

Aiming at the low precision and poor flexibility of traditional filtering algorithms, an adaptive Kalman Filtering algorithm with LSTM correction is proposed to improve the fusion accuracy of IMU and TDoA data. The coordinate correction system proposed in this paper consists of IMU unit, TDoA unit, adaptive Kalman Filter and LSTM model, as shown in Figure 1. The IMU unit is used to collect motion state information of the carrier, including a gyroscope, a magnetometer, and an accelerometer. The TDoA unit is used to acquire TDoA positioning data. Adaptive Kalman Filter is used for Filtering. The LSTM model is used for carrier trajectory correction. 


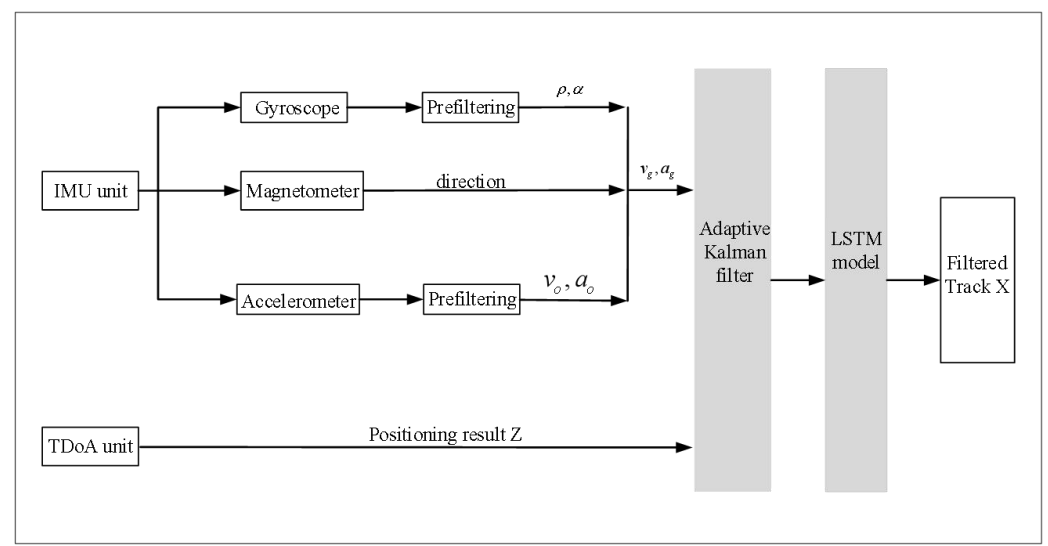

Fig. 1. System model

The fusion localization processes are as follows.

1) Data preprocessing. This process obtains three kinds of data which are gyroscope, accelerometer and magnetometer of IMU inertial measurement unit. It converts the local coordinate system of IMU inertial measurement unit into geographic coordinate system, and get the speed of carrier in geographic coordinate system and the value of the acceleration;

2) Adaptive Kalman Filtering. It combines the IMU and TDoA data by adopting the adaptive Kalman Filter algorithm to improve the data accuracy;

3) Trajectory processing with LSTM model. The LSTM model is used to smooth the latitude and longitude data in geographic coordinate to reduce the glitch or abnormal point of TDOA positioning data.

\section{Integration positioning mechanism}

\subsection{Data preprocessing}

Accelerometers, gyroscopes, and magnetometers are included in the IMU. Since the sensor tag can be installed at different positions on the research object, when it is involved in the work, the direction of the 3-axis gyroscope is not clear. Therefore, it is necessary to calculate the rotation angle by using the gyroscope meter to correct the accelerometer data to realize the above data fusion. The relationship between the coordinate system of the carrier and the geographic coordinate is shown in Figure 2.

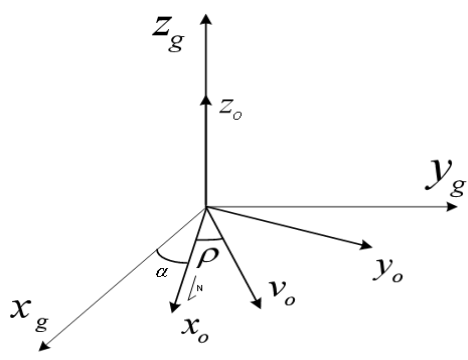

Fig. 2. Carrier coordinate system and geographic coordinate system conversion 
$x_{g} 、 y_{g}$ and $z_{g}$ is the representation of the geographic coordinate system, $x_{0} 、 y_{n}$ and $z_{n}$ means the carrier coordinate system. The angle between the velocity of the carrier motion and the north direction is $\rho$, and the angle between the carrier coordinate system and the geographic coordinate system is $\alpha, v_{o}$ is the motion velocity of the carrier in the carrier coordinate system.

IMU uses the magnetometer to determine the north direction of the carrier coordinate system, and then use the accelerometer to calculate the carrier speed. Then we take the gyroscope to calculate the angle $\rho$ between the carrier speed and the carrier coordinate system. The above information is used to calculate the velocity and acceleration components of the carrier in the geographic location coordinate. This paper considers the motion of the carrier in a planar scene. Therefore, according to the Weinberg expression ${ }^{[4]}$, it is assumed that the step size is proportional to the acceleration change of the traveling process, and the carrier step is as in equation (1).

$$
d=\delta \sqrt[4]{A_{\max }-A_{\min }}
$$

$A_{\max }$ and $A_{\min }$ respectively means the maximum and minimum of the acceleration within the step, while $\delta$ is a scaling factor to handle different walking behaviors and step lengths. The speed of the carrier is then calculated from the single-step duration of the carrier, as in equation (2)

$$
v=d\left(t_{2}-t_{1}\right)
$$

Since the inertial measurement unit can be freely mounted on the carrier, it is not clear when the intervention is in the direction of the carrier. Therefore, the rotation angle must be calculated by the accelerometer to correct the rotation angle of the gyroscope. The direction of the instantaneous velocity is $v_{o}$ and the angle between the $x_{o}$-axis of the carrier coordinate system. Due to the instantaneous angular velocity measured by the gyroscope is discrete, the current instantaneous velocity is the cumulative sum from the initial moment to the current moment, as shown in equation (3).

$$
\rho=\sum_{0}^{T} w(t) d t
$$

Given the angles $\alpha$ and $\rho$, the velocity $v_{g}$ of the carrier relative to the geographic coordinate system can be obtained, as defined in equation (4).

$$
\begin{aligned}
& v_{x, g}=v_{x, o} \times \cos \alpha=v_{o} \times \cos \rho \times \cos \alpha \\
& v_{y, g}=v_{y, o} \times \sin \alpha=v_{o} \times \sin \rho \times \sin \alpha
\end{aligned}
$$

According to the acceleration measured by the accelerometer, the acceleration $a_{g}$ of the carrier relative to the geographic coordinate system can be obtained, as shown in equation (5).

$$
\begin{aligned}
& a_{x, g}=a_{x, o} \times \cos \alpha \\
& a_{y, g}=a_{y, o} \times \sin \alpha
\end{aligned}
$$


Therefore, after data preprocessing of the IMU inertial measurement unit, the velocity and acceleration components of the carrier relative to the geographic coordinate system can be obtained, and the TDoA measurement results can be corrected for fusion localization.

\subsection{Adaptive Kalman Filter}

The Kalman Filter algorithm uses the linear system state equation to estimate the state of the system via the input and output of the system. The main process is represented by the equation of state and the observation equation, such as equations (6) and (7).

$$
\begin{aligned}
& X_{k}=A_{k, k-1} X_{k-1}+w_{k} \quad w_{k} \sim N(0, Q) \\
& Z_{k}=H X_{k}+v_{k} \quad v_{k} \sim N(0, R)
\end{aligned}
$$

Where $X$ is the state vector, $A_{k \cdot k-1}$ represents the transition matrix from the $k-1$ time state to the $k$ time state, and $w_{k}$ is the state noise matrix, obeying a Gaussian distribution with the mean of 0 and the variance of $Q . Z$ is the observation vector, $H$ is the mapping relationship between the state vector and the observation vector at time $k$, and $v_{k}$ is the observation matrix at time $k$, following a normal distribution with a mean of 0 and a variance of $R$.

Kalman Filter is a recursive solution process. In the case where the measured value of $k$ time has been measured, the state value update is mainly divided into two stages, namely the prediction stage and the update stage. In the prediction stage, the demand has the predicted probability with the mean $\bar{\mu}_{t}$ of the distribution $P\left(X_{t} \mid \mathrm{Z}_{1: t-1}\right) \sim \mathrm{N}\left(\bar{\mu}_{t}, \bar{\Sigma}_{t}\right)$ and the variance $\bar{\Sigma}_{t}$. In the update phase, the demand has to update the mean $\hat{\mu}_{t}$ and $\hat{\Sigma}_{t}$ variance of the probability distribution $P\left(X_{t} \mid Z_{1: t}\right)=N\left(\hat{\mu}_{t}, \hat{\Sigma}_{t}\right)$.

(1) In the prediction phase, the mean $\bar{\mu}_{t}$ expression is as in equation (8).

$$
\bar{\mu}_{t}=E\left[X_{t} \mid Z_{1: t-1}\right]=A \widehat{\mu}_{t-1}
$$

The variance $\bar{\Sigma}_{t}$ expression of the predicted probability is expressed by equation (9).

$$
\bar{\Sigma}_{t}=E\left[\left(\Delta X_{t}\right)\left(\Delta X_{t}\right)^{T}\right]=A \hat{\Sigma}_{t-1} A^{T}+Q
$$

Where $\Delta X_{t}$ is the amount of change in the state of the carrier in a single step.

(2) In the update phase, the intermediate variable Kalman gain $K$ is first calculated, as in equation (10).

$$
K=\bar{\Sigma}_{t} H^{T}\left(H \bar{\Sigma}_{t} H^{T}+R_{t}\right)^{-1}
$$

The expression of the mean $\hat{\mu}_{t}$ of the update probability is expressed by equation (11).

$$
\hat{\mu}_{t}=\bar{\mu}_{t}+K\left(Z_{t}-H \bar{\mu}_{t}\right)
$$

The expression of the variance $\hat{\Sigma}_{t}$ of the update probability is expressed by the equation (12).

$$
\hat{\Sigma}_{t}=(I-K H) \bar{\Sigma}_{t}
$$

The updated probability mean $\hat{\mu}_{t}$ is the updated state. 
The traditional Kalman Filter algorithm is just applicable to linear control systems with a priori statistical properties of known noise. However, in the process of carrier motion, due to the complexity of the actual system, it is very difficult to obtain the system noise $Q$ and the measurement noise $R$ of the motion model system. Due to the time-varying and random nature of the actual situation, the statistical properties of the noise may be unknown and timevarying, and the prior data is often lost due to changes in the situation. The Sage-Husa Adaptive Kalman Filter algorithm can solve this kind of problem ${ }^{[10-12]}$. When using the observation data for update filtering, the filter itself determines whether the system dynamics model changes. That is, the system noise $Q$ or the measurement noise $R$ is estimated online according to the measured value and the updated value of the system, the change of the noise is tracked in real time, and the parameters of the filter are corrected to improve the filtering precision.

During the motion of the carrier, the observation vector of the TDoA positioning output is $Z_{1}=[x, y]^{T}$, and the observation vector after the data preprocessing by the IMU system is $Z_{2}=\left[v_{x}, v_{v}, a_{x}, a_{v}\right]^{T}$. By integrating the IMU and TDoA data, the TDoA data is modified and the observation vector $Z=\left[Z_{1}, Z_{2}\right]^{T}$ of the system is obtained and state vector $X_{k}=\left[x_{k}, y_{k}, v_{x . k}, v_{v . k}, a_{x . k}, a_{v . k}\right]$. When the carrier single-step motion time is $\Delta t$, it is obtained by a kinematic formula, and the state transition matrix is as shown in equation (13).

$$
A_{k, k-1}=\left[\begin{array}{cccccc}
1 & 0 & \Delta t & 0 & \frac{\Delta t^{2}}{2} & 0 \\
0 & 1 & 0 & \Delta t & 0 & \frac{\Delta t^{2}}{2} \\
0 & 0 & 1 & 0 & \Delta t & 0 \\
0 & 0 & 0 & 1 & 0 & \Delta t \\
0 & 0 & 0 & 0 & 1 & 0 \\
0 & 0 & 0 & 0 & 0 & 1
\end{array}\right]
$$

In the motion of the carrier, both velocity and acceleration are variables, meaning that the value obeys the Gaussian distribution, and the variance $Q$ of the state noise matrix is as shown in equation (14). 


$$
Q=\left[\begin{array}{cccc}
\Delta t & 0 & \frac{\Delta t^{2}}{2} & 0 \\
0 & \Delta t & 0 & \frac{\Delta t^{2}}{2} \\
1 & 0 & \frac{\Delta t^{2}}{2} & 0 \\
0 & 1 & 0 & \frac{\Delta t^{2}}{2} \\
0 & 0 & 1 & 0 \\
0 & 0 & 0 & 1
\end{array}\right]\left[\begin{array}{c}
\sigma_{v x, k-1} \\
\sigma_{v y, k-1} \\
\sigma_{a x, k-1} \\
\sigma_{a y, k-1}
\end{array}\right]
$$

Where $\sigma_{v x . k-1}, \sigma_{v v . k-1}, \sigma_{\text {ax.k-1 }}$ and $\sigma_{a v . k-1}$ respectively represents the velocity and acceleration noise variance of the carrier in the geographic coordinate axis. In equation (8), the observation matrix $H=I, R$ is the measurement noise covariance matrix, and its value is correlated with the measurement and measured by experimental means. After setting the initial values of $Q$ and $R$, they are substituted into the Adaptive Kalman Filter algorithm, and are continuously updated to improve the overall trajectory correction accuracy.

\subsection{Track correcting with LSTM model}

The LSTM network is a time recurrent neural network that is an enhancement to the RNN neural network and is suitable for processing and predicting time series ${ }^{[13-16]}$. In this paper, the LSTM network model is used to modify the carrier trajectory.

1) Recurrent Neural Network RNN

The difference between the structure of RNN and standard neuron networks is that it has a recursive structure that can transmit information from the last state to the current state, as shown in Figure 3. When the input is time series, it can be extended to a series of interconnected standard neuron networks.

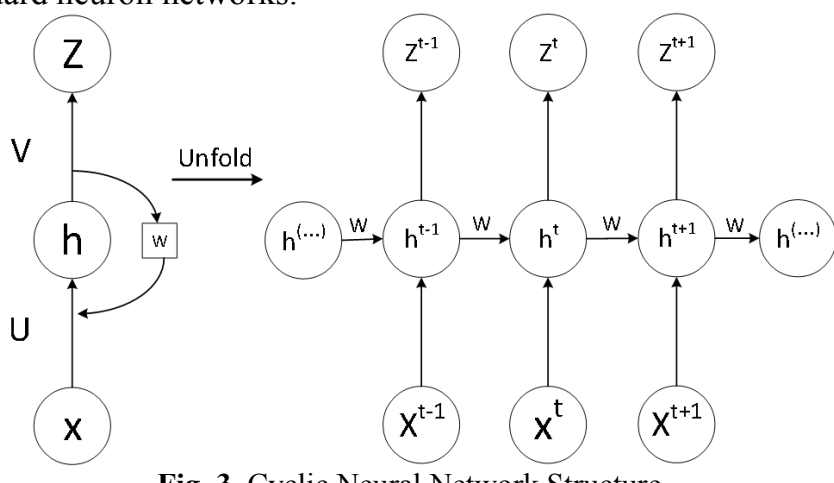

Fig. 3. Cyclic Neural Network Structure

As shown in Figure 3, each node represents a single layer neural network at a single point in time. The weight from the input layer to the hidden layer is labeled $U$, the hidden layer to its own weight is labeled $W$, and the hidden layer to output layer is labeled as $V$. These 
weights will be reused in each sequence. However, for traditional RNNs, some of the information will be lost during each feedback process. When the time reaches a certain point, the initial information will be degraded and the gradient disappears, so the RNN loses its ability to remember for a long time.

2) Long Short-Term Memory LSTM

The difference between the LSTM network and the standard RNN is that the structure of the hidden unit of the RNN is replaced by the memory module, avoiding the disappearance of the gradient of the regular RNN. The memory unit is a Cell structure, and three doors are placed in one Cell, which are an input gate, a forgetting gate, and an output gate. A message enters the LSTM network through the input gate, which can be judged according to the rules. Only information that conforms to the algorithm's authentication will be left and output via the output gate. The mismatched information is forgotten through the Forgotten Gate. It can be described as Figure 4.

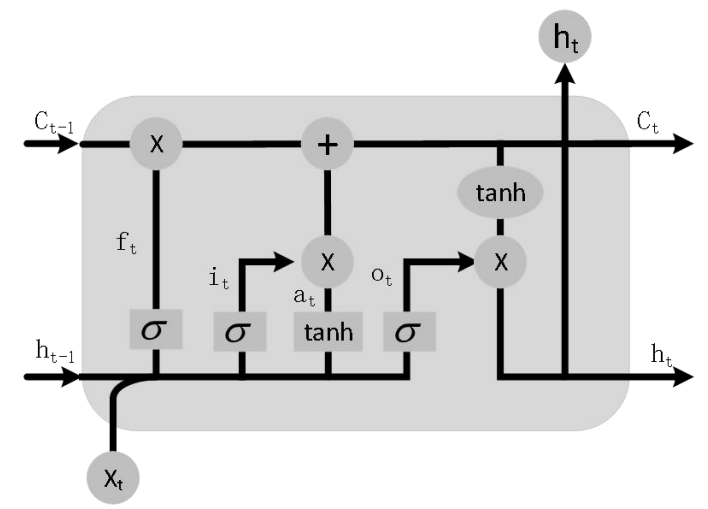

Fig. 4. LSTM network Cell structure

In the process of updating in the Cell, first though updating the output of the forgetting gate, the part of the historical motion trajectory of the carrier is forgotten, and the time period long from the current time is less correlated with the current path prediction. However, the carrier motion trajectory in the time period close to the current time is highly correlated with the trajectory of the carrier at the next time, and thus the partial information remains. The forgetting gate output expression is as shown in equation (15).

$$
f_{t}=\sigma\left(W_{f} \cdot h_{t-1}+U_{f} x_{t}+b_{f}\right)
$$

After that, the output of the input gate is updated. This part determines the information learned by the system. When the new coordinate track point of the carrier is input, the Cell analyzes the current trajectory and learns the trend of the recent historical trajectory, so as to correct the carrier trajectory value later. The expression of the input gate is as shown in equation (16).

$$
\begin{aligned}
& i_{t}=\sigma\left(W_{i} \cdot h_{t-1}+U_{i} x_{t}+b_{i}\right) \\
& a_{t}=\tanh \left(W_{C} \cdot h_{t-1}+U_{a} x_{t}+b_{a}\right)
\end{aligned}
$$

The results of both the forgetting gate and the input gate will act on the cell state $C_{t}$, as in equation (17). 


$$
C_{t}=f_{t} \odot C_{t-1}+i_{t} \odot a_{t}
$$

Where $\odot$ represents the Hadamard product of the matrix.

Next, update the output gate and decide to enter the state of the next stage of the Cell message, as in formula (18).

$$
\begin{aligned}
& o_{t}=\sigma\left(W_{o} h_{t-1}+U_{o} x_{t}+b_{o}\right) \\
& h_{t}=o_{t} \odot \tanh \left(C_{t}\right)
\end{aligned}
$$

Finally, the index of the current sequence is updated and the predicted value of the carrier at the next stage position is output, as in equation (19).

$$
Z_{t}=\sigma\left(V h_{t}+c\right)
$$

When the LSTM model predicts the value of the next coordinate from the historical trajectory coordinates, it is weighted and averaged with the trajectory coordinate value of the Adaptive Kalman Filter at the next moment. The weight is obtained by the accuracy ratio of the model prediction and the accuracy ratio of the Adaptive Kalman Filter algorithm, and then the smoothing of the carrier trajectory is realized.

\section{Result}

In order to evaluate the proposed trajectory correction algorithm, different walking tests were performed in an empty corridor of size $25 \mathrm{~m} \times 8 \mathrm{~m}$. The eight TDoA anchors in the experiment were placed in a rectangular shape. In order to achieve communication between different anchor points and the central server, each anchor point is equipped with Ethernet. And the central server will provide TDoA measurements for the positioning engine ${ }^{[17-19]}$. To ensure that the user's different measurement unit positions worked as well as the different walking behaviors, the three people test with three different measuring unit positions, one placed in the hand, one attached to the belt, and the last tester places the measuring unit in the pocket of the shirt. Since the tester moves up and down during the movement, and the measuring device causes the system noise and measurement noise to disobey the Gaussian distribution due to factors such as the use time, so the noise constantly changes with time. By comparing Kalman filter, adaptive Kalman filter and adaptive Kalman filter with LSTM correction to the filtering accuracy of the motion trajectory, the proposed system is evaluated. The positioning index is the mean value of the error vector 1 norm obtained by the difference between the filtered value of the undetermined target position coordinate and the real value of the undetermined target position coordinate, that is the relative coordinate error.

Figure 5 shows the results of a normal test in which the measuring unit is held in the hand by the tester. The black curve represents the true path trajectory, while the green, blue, and red curves represent the trajectory filter values of Kalman filtering, adaptive Kalman filtering, and adaptive Kalman filtering with LSTM correction, respectively. After calculation, the relative coordinate error of the measured value of the carrier coordinates relative to the real walking trajectory is $0.8532 \mathrm{~m}$, the relative coordinate error after Kalman filtering is $0.6214 \mathrm{~m}$, and the relative coordinate error after adaptive Kalman filtering is $0.4535 \mathrm{~m}$, the relative coordinate error of the LSTM modified adaptive Kalman filter algorithm is $0.2732 \mathrm{~m}$. 


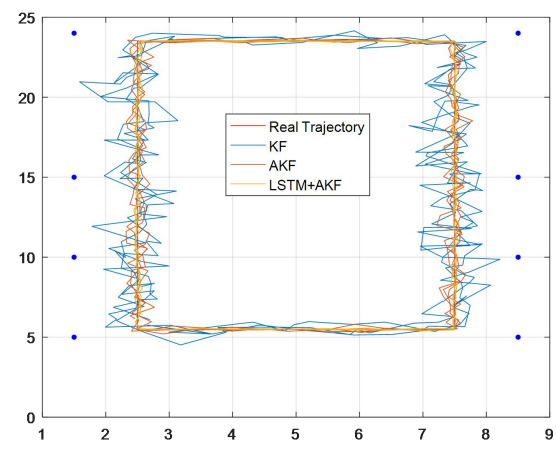

Fig. 5. rectangular path route filtering

Figure 6 shows a test case in which the measuring unit is placed in the tester's shirt pocket. In this test, the proposed model has poor accuracy in estimating the position at the initial stage, but after a short time, the filter trajectory converges. The longer the tester spends walking, the better performance will be in the filter. It can be seen from Fig. 6 that the proposed adaptive Kalman filter algorithm with LSTM correction can achieve convergence faster than the Kalman filter algorithm. After calculation, the relative coordinate error of the measured value of the carrier coordinates relative to the real walking trajectory is $0.7562 \mathrm{~m}$, the relative coordinate error after Kalman filtering is $0.5234 \mathrm{~m}$, and the relative coordinate error after adaptive Kalman filtering is $0.4575 \mathrm{~m}$, the relative coordinate error of the LSTM modified adaptive Kalman filter algorithm is $0.3632 \mathrm{~m}$.

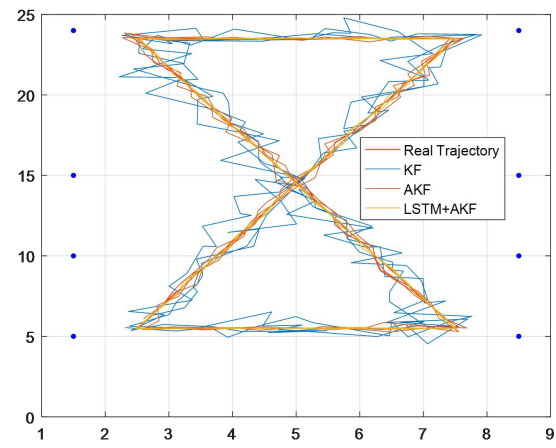

Fig. 6. cross track path filtering

The cumulative distribution function of the trajectory filter error of all the testers in Fig. 7 shows that the proposed adaptive Kalman filter algorithm with LSTM correction performs well. In $90 \%$ of cases, the error of the adaptive Kalman filter with LSTM correction is $1.45 \mathrm{~m}$ or lower, while the error of the adaptive Kalman filter algorithm is $1.72 \mathrm{~m}$. The error of the traditional Kalman filter algorithm is $2.42 \mathrm{~m}$. 


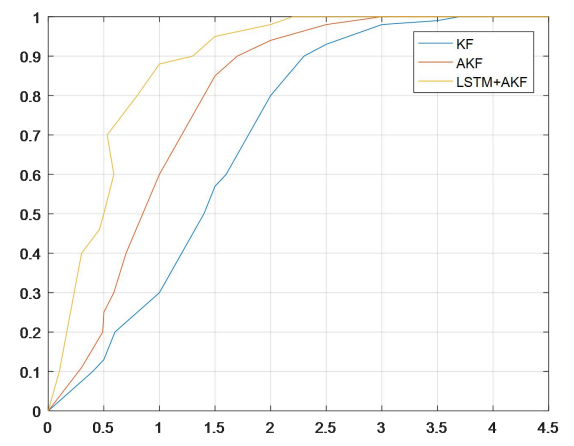

Fig. 7. Cumulative error distribution function

It can be obtained from experiments that the adaptive Kalman filter algorithm with LSTM correction can correct the measurement target trajectory well, thus improving the positioning accuracy.

\section{Conclusion}

In order to solve the problem that the system state noise and measurement noise are constantly changing during the motion, this paper proposes an adaptive fusion positioning mechanism of TDoA and IMU data with LSTM correction. Firstly, the IMU data is transformed into the geographic coordinate system, then the adaptive Kalman filter algorithm is used to fuse the inertial measurement unit with the TDoA result, and then the trajectory prediction result of the LSTM model is combined to correct the trajectory of the carrier. The simulation results show that the proposed correction mechanism can improve the trajectory correction accuracy under non-Gaussian noise interference and has good scalability. Therefore, in the subsequent research, the positioning solution of the carrier in the non-Gaussian nonlinear motion system will be continuously studied, the complexity of the model will be reduced to improve the positioning accuracy of the fusion solution and reduce the system energy consumption.

\section{ACKNOWLEDGMENTS}

This work is supported by State Grid Corporation of China headquarters science and technology project(546816180001).

\section{References}

[1] Choi M-S, Jang B. “An Accurate Fingerprinting based Indoor Positioning Algorithm.” International Journal of Applied Engineering Research, vol. 12, no. 1, pp. 86-90. 2017.

[2] M. I. AlHajri et al., "Hybrid RSS-DOA technique for enhanced WSN localization in a correlated environment," 2015 International Conference on Information and Communication Technology Research (ICTRC), Abu Dhabi, 2015, pp. 238-241. 
[3] J. Lategahn, M. Müller and C. Röhrig, "Extended Kalman filter for a low cost TDoA/IMU pedestrian localization system," 2014 11th Workshop on Positioning, Navigation and Communication (WPNC), Dresden, 2014, pp. 1-6.

[4] Z. Zeng, S. Liu and L. Wang, "A novel NLOS mitigation approach for TDOA based on IMU measurements," 2018 IEEE Wireless Communications and Networking Conference (WCNC), Barcelona, 2018, pp. 1-6.

[5] A. Lewandowski and C. Wietfeld, "A comprehensive approach for optimizing ToAlocalization in harsh industrial environments," IEEE/ION Position, Location and Navigation Symposium, Indian Wells, CA, 2010, pp. 516-525.

[6] D. Ayllón, H. A. Sánchez-Hevia, R. Gil-Pita, M. U. Manso and M. R. Zurera, "Indoor Blind Localization of Smartphones by Means of Sensor Data Fusion," in IEEE Transactions on Instrumentation and Measurement, vol. 65, no. 4, pp. 783-794, April 2016.

[7] M. Kolakowski, "Utilizing acceleration measurements to improve TDOA based localization," 2017 Signal Processing Symposium (SPSympo), Jachranka, 2017, pp. 1-4.

[8] Gravina R, Alinia P, Ghasemzadeh H, et al. "Multi-sensor fusion in body sensor networks: State-of-the-art and research challenges." Information Fusion, vol. 35, pp. 68-80, 2017.

[9] Fujii K. "Extended kalman filter." Refernce Manual, 2013.

[10] Isaksson A . "Identification of Time Varying Systems Through Adaptive Kalman Filtering." 1987.

[11] D. Jwo and S. Wang, "Adaptive Fuzzy Strong Tracking Extended Kalman Filtering for GPS Navigation," in IEEE Sensors Journal, vol. 7, no. 5, pp. 778-789, May 2007.

[12] A. A. Girgis and D. G. Hart, "Implementation of Kalman and adaptive Kalman filtering algorithms for digital distance protection on a vector signal processor," in IEEE Transactions on Power Delivery, vol. 4, no. 1, pp. 141-156, Jan. 1989.

[13] Lizhe Liu, W. Chen and Guitao Cao, "Prediction of neonatal amplitude-integrated EEG based on LSTM method," 2016 IEEE International Conference on Bioinformatics and Biomedicine (BIBM), Shenzhen, 2016, pp. 497-500.

[14] Z. Zhao, W. Chen, X. Wu, P. C. Y. Chen and J. Liu, "LSTM network: a deep learning approach for short-term traffic forecast," in IET Intelligent Transport Systems, vol. 11, no. 2, pp. 68-75, 32017.

[15] T. Ergen and S. S. Kozat, "Online Training of LSTM Networks in Distributed Systems for Variable Length Data Sequences," in IEEE Transactions on Neural Networks and Learning Systems, vol. 29, no. 10, pp. 5159-5165, Oct. 2018.

[16] B. Cheng, X. Xu, Y. Zeng, J. Ren and S. Jung, "Pedestrian trajectory prediction via the Social-Grid LSTM model," in The Journal of Engineering, vol. 2018, no. 16, pp. 1468-1474, 112018.

[17] Huang, Baoqi, L.Xie, and Z.Yang, "TDOA-Based Source Localization With DistanceDependent Noises," in IEEE Transactions on Wireless Communications, vol. 14, no. 1, pp. 468-480, Jan. 2015.

[18] Chan, Yiu Tong, and K. C. Ho. "Joint time-scale and TDOA estimation: analysis and fast approximation," in IEEE Transactions on Signal Processing, vol. 53, no. 8, pp. 2625 2634, Aug. 2005.

[19] Meng W , Huang B , Gao G. "A novel approach to NLOS identification in sensor localization," The 26th Chinese Control and Decision Conference (2014 CCDC), Changsha, 2014, pp. 876-880. 